\title{
The Use of Steroids in Spinal Cord Injuries: Origins of a Controversy
}

\author{
Eric Dennis C. Legaspi
}

Division of Neurological Surgery, Department of Neurosciences, College of Medicine and Philippine General Hospital, University of the Philippines Manila

\section{Introduction}

The use of steroids in the treatment of acute spinal cord injuries is a wide-spread practice that is often considered a local standard of care. It has even occasionally made its way to being a legal standard of practice. For instance, in 1998, a team of physicians was found liable for inadequately addressing the spinal cord injury of an infant under their care. ${ }^{1}$ During a high forceps delivery, the head of the infant had been inadvertently rotated 180 degrees rendering the child quadriplegic and ventilator dependent. Sixty percent of the penalty imposed on the medical team was assigned to the neonatologists who attended the child after the injury had occurred, in part because:

"Two expert witnesses testified that had steroids been administered within an 8 hour window post-injury, there would have been a 95\% probability of significant improvement in the infant's ability to breathe and move her arms." 2

Evidently, the use of steroids in acute cord injury had become a standard of the care expected by the local community. The Medical Student Curriculum in Neurosurgery currently recommended by the Congress of Neurological Surgeons similarly states that:

"...Patients with spinal cord injury who were treated with methylprednisolone within 8 hours of injury had significantly greater improvement in their neurologic function...than those given a placebo."

"...Patients with acute spinal cord injury who receive methylprednisolone within 3 hours of their injury should be maintained on the treatment regimen for 24 hours. When methylprednisolone is initiated 3 to 8 hours after the injury patients should be maintained on steroid treatment for 48 hours..."3

The authoritative Cochrane Database also advocates the use of high dose methylprednisolone for acute spinal cord injury, stating it is:

"... the only pharmacologic therapy shown to have efficacy in a phase three randomized trial..." 4,5

So is the use of high dose steroids in acute spinal cord injury actually warranted? Current medical literature has

\footnotetext{
Corresponding author: Eric Dennis C. Legaspi, MD

Division of Neurological Surgery

Department of Neurosciences

Philippine General Hospital

Taft Avenue, Ermita, Manila 1000 Philippines

Telephone: +6325242338

Email: eclegaspi@gmail.com
}

cast considerable doubt on the effectiveness of this practice, which has been abandoned in many places. Since it still remains one of the prevalent measures being used in the Philippines today, there is a need to look further into the basic rationale behind its use, and the history behind its rise to prominence as well as its fall from grace.

\section{The Case for Steroid Use - The NASCIS Trials}

Although the use of steroids in treating acute spinal cord injury had been a long standing practice, its main scientific backing came from the data generated by the National Acute Spinal Cord Injury Study (NASCIS), which was reported in a series of articles. ${ }^{6-11}$ NASCIS was a multicentered, double-blinded, randomized trial that examined the use of three drugs - methylprednisolone, tirilazad, and naloxone - in the treatment of acute spinal cord injury. It was conducted under a team of physicians from the Yale University School of Medicine, headed by Dr. Michael Bracken, an eminent epidemiologist, and was considered a landmark study which could serve as a model for the conduct of clinical trials. It is no exaggeration to say that rationale for the use of methylprednisolone in acute spinal cord injury has stood on the pillars of the NASCIS findings. For example, the earlier mentioned Congress of Neurological Surgeons medical school curriculum ${ }^{3}$ is a reiteration of the NASCIS recommendations, and both the 2001 and 2012 Cochrane reviews endorsing the use of methylprednisolone ${ }^{4,5}$ were written by the NASCIS lead author, Dr. Bracken. The current TraumaBank Informational Repository ${ }^{12}$ lists only 5 prospective randomized trials dealing with the topic, 3 of which were generated by NASCIS. NASCIS therefore serves as a good place to begin an examination of the medical evidence for the use of steroids.

\section{NASCIS I}

Prior to NASCIS, many animal studies had shown steroids to have a protective effect on the acutely injured spinal cord. Black and Markowitz ${ }^{13}$ showed steroids to be superior to local hypothermia in alleviating the effects of experimental cord injury in monkeys. They were also shown to decrease the histopathological and chemical effects of cord contusions in cats $^{14,15}$ and to improve recovery in experimentally injured ferrets. ${ }^{16}$ Since steroids, especially dexamethasone, were already in wide clinical use to control the edema caused by neoplasms and infections, a trial on 
steroid use for acute injuries of the human spinal cord was almost inevitable.

NASCIS I was officially reported in $1984,{ }^{6}$ with a one year follow up reported in 1985.7 Three-hundred and thirty patients with acute spinal cord injury were randomized into two groups who then received methylprednisolone at either a high dose (1000 mg intravenous bolus on admission, then $250 \mathrm{mg}$ i.v. every 6 hours for 10 days) or a standard dose (100 mg i.v. bolus on admission then $25 \mathrm{mg}$ i.v. every 6 hours for 10 days). Spinal cord recovery was then measured at 6 weeks, 6 months, and one year after injury, in terms of motor function, pinprick response, and touch sensation. NASCIS chose methlyprednisolone rather than the more readily available dexamethasone as the steroid for testing. This was because methylprednisolone did not react with anti-convulsants, passed more rapidly through cell membranes, and had been shown to be more effective in inhibiting neutropenic responses to activated component something which was felt to be a key mechanism in preventing neuronal injury.

NASCIS I failed to show any significant difference in outcome between the two test groups. In fact, early case fatalities were greater under the high dose protocol, as was the incidence of infection of both trauma and surgical sites, with the difference failing to meet statistical significance.

One remarkable feature of the NASCIS I experimental design though, was the failure to include a placebo control. This, the researchers explained, was a reaction to the prevailing social atmosphere.

"In this study, we were not able to compare the high dose steroid against placebo. At the time the study was established existing wide-spread use of steroids and concern over potential medical malpractice suits if steroids were withheld dampened enthusiasm for a placebo study arm."7

The lack of a placebo control coupled with the failure of the data to demonstrate a significant difference between the two drug regimens cast some doubt on the over-all efficacy of methylprednisolone. The researchers felt though that further investigation was warranted. Two possibilities were raised to explain the lack of a different outcome between the standard and high dose groups. One was that the standard dose had already conferred a maximal benefit:

"Any interpretation of the lack of a treatment effect observed must include the possibility that both doses of steroid were of equal benefit..." 6

"This study does not necessarily suggest that methylprednisolone is inefficacious...It is possible that the standard methylprednisolone dose improves neurological recovery and that the tenfold larger dose offers no additional improvement..."7

The alternative possibility raised was that even the high dose regimen was actually too low.

"It is possible that the high dose of methylprednisolone used in this study did not reach therapeutic levels..." 7
It was this latter reasoning that was eventually followed in the design of the NASCIS trials that followed.

\section{NASCIS III}

As the design and results of the NASCIS III trial rely heavily on the results of NASCIS II, it is more convenient to discuss them first.

The third NASCIS trial was reported in $1997^{10}$ with a one year follow up reported in 1998. ${ }^{11}$ NASCIS III was again a randomized, double-blind study, this time dividing the study population into three experimental groups, then evaluating neurologic function on admission, then 6 weeks, 6 months, and 1 year after treatment. Two drugs were investigated in this study - methylprednisone and tirilazad mesylate, a potent inhibitor of lipid peroxidation. In the 24MP group, 166 patients received methylprednisolone according to the protocol that had been established in NASCIS II (30 mg/kg loading dose, then $5.4 \mathrm{mg} / \mathrm{kg} / \mathrm{hour}$ over the next 23 hours). In the 48MP group, 166 patients received an initial dose of methylprednisolone according to the $24 \mathrm{MP}$ protocol, but administration of $5.4 \mathrm{mg} / \mathrm{kg} / \mathrm{hour}$ was extended until the 48th hour after admission. The third group was the 48TM where 167 patients received a loading bolus of tirilazad mesylate at a dose of $2.5 \mathrm{mg} / \mathrm{kg}$, then the same dose every 6 hours for 48 hours.

Simply stated, NASCIS III showed that patients treated within 3 hours of the spinal cord injury recovered equally well in all three groups. If the start of treatment was delayed by 3 to 8 hours, then the $24 \mathrm{MP}$ protocol showed a decline in efficacy while the $48 \mathrm{MP}$ group did not. In general patients receiving tirilazad under the $48 \mathrm{TM}$ protocol recovered equally as well as the patients under the $24 \mathrm{MP}$ regimen.

Significantly though, NASCIS III again did not include a control group, instead randomizing all the admitted patients into one of three treatment arms (24MP, 48MP, and 48TM). The NASCIS group later justified this, citing:

"... a large body of literature suggesting that it is unethical to use placebos when an effective therapy has already been documented. The NASCIS neurosurgeons in NASCIS III, having previously documented an effective therapy in NASCIS II, quite properly rejected the notion of giving patients placebos in the new trial..."17

The researchers regarded the data generated by NASCIS II as being sufficient to prove the benefits of methylprednisolone over a placebo, stating again:

"Patients treated with MP within 8 hours of injury experienced significantly improved neurologic function over placebo-treated patients 6 weeks, 6 months, and 1 year postinjury...". ${ }^{11}$

\section{NASCIS II}

NASCIS II was reported in $1990^{8}$ with a one year follow up reported in $1992 .{ }^{9}$ It was the only phase of the NASCIS 
studies that included a comparison with a placebo control, and as such deserves the closest scrutiny.

Like the other NASCIS trials, NASCIS II was a randomized, double-blind study which covered 487 patients with acute spinal cord injury, $95 \%$ of whom were treated within 14 hours of injury. Three randomized study groups were formed. One hundred sixty-two patients received a loading dose of $30 \mathrm{mg} / \mathrm{kg}$ of methylprednisolone on admission followed by an infusion of $5.4 \mathrm{mg} / \mathrm{kg} /$ hour over the next 23 hours. (What would later come to be known as the $24 \mathrm{MP}$ protocol.) One hundred fifty-four patients received a loading dose of $5.4 \mathrm{mg} / \mathrm{kg}$ of naloxone followed by an infusion of $4.0 \mathrm{mg} / \mathrm{kg} /$ hour over the next 23 hours. The control group of 171 patients received a placebo delivered in "look-alike" packaging. All patients were evaluated on admission, then again 6 weeks, 6 months, and 1 year after admission. Scores were assigned for motor function, pinprick sensation, and light touch. ${ }^{1}$

To evaluate the patients' motor function, 14 muscle segments were tested and graded according to the scale of

0 No contraction

1 Reduced contraction

2 Movement without anti-gravity function

3 Some anti-gravity function

4 Reduced movement against resistance

5 Normal function

Scores ranged from 70 (normal at all levels) to 0 (no contraction at all levels) and were scored for both sides of the body.

Pinprick and light touch were examined for the 29 dermatomes from C2 to S5, and were scored as:

1 absent

2 decreased

3 normal

Scores ranged from 29 (absent at all levels) to 87 (normal at all levels).

The results of NASCIS II showed no significant benefit among the patients who had received naloxone. The patients who had received methylprednisolone within 8 hours of sustaining their cord injury, however, showed a clear advantage over those who had only received a placebo. Six months after injury, motor function in the methylprednisolone group showed a 16.0 point improvement, as compared to 11.2 points in the placebo group $(p=0.03)$. Pinprick improved 11.4 points as compared to 6.6 points $(p=0.02)$, and light touch improved 8.9 points as compared to $4.3(\mathrm{p}=0.03) .{ }^{8}$ Furthermore, the use of methylprednisolone did not seem to be associated with increased complications. The study group wrote:

"Even if the small increases in wound infection and gastrointestinal bleeding found in the methylprednisolone treated patients were truly related to treatment (in this study they cannot be distinguished from chance) they are manageable conditions and the risk associated with them would be well worth the potential therapeutic benefits..." 9

The benefits of methylprednisolone persisted to the reevaluation 1 year after injury, with significant improvement seen in motor recovery, as well as in touch and pinprick sensation. ${ }^{9}$

With NASCIS II, it seemed that the basis for recommending methylprednisolone as a safe, effective drug in the treatment of acute spinal cord injury had been firmly laid.

\section{Reactions to NASCIS II}

Yet the results of NASCIS II were met with some resistance from the medical community. Part of the criticism arose because the results of the drug trials were announced to the popular press before they were published in a peerreviewed journal. The expressed rationale for this unusual move was the need for rapid dissemination of knowledge and therapy. It was felt however that the early publicity given to the trials may have led to data over-simplification and misinterpretation. There was an over-all failure to recognize exclusion criteria or appropriate legal ramifications. By not communicating with the appropriate physicians, rational use of the drug was hampered. ${ }^{18}$ This tendency of the NASCIS group to bypass peer scrutiny in favor of general public approval was noted by other authors as well. As late as 2000, Coleman complained:

"... even 9 years after NASCIS II, the primary data have not been made public. The reporting of the NASCIS studies has fallen far short of the guidelines of the ICH/FDA and of the EvidenceBased Medicine Group...There has been no public process of validation..."19

In fact, data as basic as the identity of the muscle groups tested for motor function was not stated in the original papers, and only released years after the conclusion of the study.

\section{Other Criticisms of NASCIS}

Other criticisms have been levelled at different aspects of the NASCIS trials.

A. The study population. The inclusion criteria, for example, defined acute spinal cord injury as:

"Any loss of sensation (pinprick or light touch) or motor function below the lesion was indicative of spinal cord trauma." 6

By this definition, even patients with mild, transient injuries were included in the analysis. As was later pointed out, up to one half of the NASCIS III sample may have been suffering from at most, a minor deficit. ${ }^{19}$ While this group of patients carries its own medical interest, clearly it is not a population at critical risk.

Also, the number of patients contributing meaningful data may not be as large as it would initially seem. The single most important finding of the NASCIS II trials would have to be that the early administration of high dose steroids 
improves motor function in patients with cord injury. Yet of the 487 patients in NASCIS II, 154 received naloxone, which proved ineffective. A further 198 had their treatment started 8 or more hours after injury, and reported little or no benefit. The actual numbers of patients with data bearing directly on the benefit of early steroid administration is the 66 patients who received the proper $24 \mathrm{MPS}$ protocol, and the 69 patients who received placebo. Even counting the cost of treating each individual, these numbers seem rather small. ${ }^{20}$

B. The motor evaluation. Considering the importance of the data on motor function, the NASCIS II researchers also made a curious decision. Data was collected for motor strength on both sides of the body.

"Expanded motor scores ranged from 0 (no contractions in any muscle) to 70 (all normal responses, and were obtained separately for the right and left sides..." 9

And then half of the data set was removed from the analysis.

"Data from the right side...were arbitrarily chosen for analysis. Earlier analysis showed that using data from the left side did not materially influence the results..."9

The decision to arbitrarily remove one half of the most critical data set was confusing. It was made even more inexplicable by the fact that the data on pinprick and light touch sensation were reported for both sides. While a follow-up statistical analysis was later provided to substantiate this claim, the long-standing refusal of the NASCIS group to release their primary data set to allow independent verification clouded the issue.

Other groups also questioned whether the change in motor scores actually constituted a clear benefit to the patient. In commenting on the improvement of motor scores of 16.0 points in the methylprednisolone group versus the 11.2 points in the placebo group, it was noted that:

"...The post-hoc analysis identified a statistically significant improvement of 5 motor points. But is this clinically significant? An improvement of 5 motor points in one muscle group will not confer any increased functionality on a spinal cord injured patient. Similarly, if spread across 5 different muscle groups will have little impact on functional ability..." 12

Of more use would have been the use of a Functional Independence Measurement (FIM) evaluation in NASCIS II to see if the patients' over-all functional capacity was enhanced by the use of steroids over mere placebo. FIM testing was actually performed in NASCIS III, but again, the failure to include a placebo control in this phase of the trial prevented a direct evaluation. Instead, the researchers compared the NASCIS III study groups to the NASCIS II groups, then stated:

"It seems highly likely therefore, that had FIM been assessed in NASCIS II, similar improvements in 24MPS compared to placebo FIM scores would have been observed..."11
C. Post hoc data analysis. Several authors also commented on the re-organization of previously collected data into new groupings after initial evaluation failed to yield satisfactory results $2,19,21$ the process of "post hoc analysis". The initial research question NASCIS II was designed to answer was whether there was a difference in acute spinal cord injury patients treated with methylprednisolone, naloxone, or placebo. Initial analysis showed no significant difference in treatment groups. It is only when the data was re-analysed that the benefits of treating before the 8 hour cut-off (and later the 3 hour cut-off recommended in NASCIS III) were reported.

Yet the treatment of the 8 hour cut-off as an all-or-none limit is neither intuitive or physiologic. A more convincing result would have shown the benefits of steroid administration to fall in a curve - that is, steroids given within 1 hour of injury would yield better results than those given 1 to 2 hours from injury, which in turn would be superior to those given 2 to 3 hours after, and so on. The failure to present this curve along with the refusal to release raw data to allow independent analysis has weakened the impact of NASCIS II. When the raw data was finally released, independent statisticians pointed out that post hoc analysis could be used to prove several illogical conclusions, among them:

- Patients with complete motor and incomplete sensory deficits did better on placebo than on 24MP2

- Patients with incomplete spinal cord injury who received placebo more than 8 hours after injury had significantly better recovery than those who received placebo within 8 hours. ${ }^{19}$

- Patients with incomplete spinal cord injury given $24 \mathrm{MP}$ within 8 hours had recovery as good as those given placebo after 8 hours. ${ }^{21}$

D. Understatement of risks. The NASCIS reports may have understated the incidence of complications arising from the administration of high dose steroids. NASCIS I for example reported:

"...wound infection differed significantly between treatments occurring 3.6 times more frequently in patients given the high dose $(p=0.01)$

"Death within 14 days of injury was 3.10 times as common in patients under the high dose protocol and 1.92 times as common within 28 days..."

"78\% increased risk of pulmonary embolus among high dose patients..."

And rather surprisingly:

"The elevated case fatality rate in the high-dose steroid patients was of such concern, especially in the absence of any evident benefit, that patient accrual into the trial was discontinued several months before the planned termination date..." ${ }^{6,7}$ 
NASCIS III also reported:

"More deaths resulted from pneumonia, respiratory distress syndrome and respiratory failure in the 48TM and 48MPS groups, a difference that may not be due to chance $(p=0.056)$ "

"...significant increases in severe pneumonia seen at 6 weeks after 48MP treatment, and the observation of possible increased mortality rates caused by pneumonia, RDS, or respiratory failure call for caution..." 10

NASCIS II showed no significant difference in the complication rates of patients administered steroids over those given a placebo. 8,9 However, another retrospective study of patients treated with the $24 \mathrm{MP}$ protocol demonstrated a significantly increased incidence of pneumonia, as well as longer ICU stays and ventilated days over those given a placebo. ${ }^{22} \mathrm{~A}$ different retrospective study showed that the use of the $24 \mathrm{MP}$ protocol was not associated with an increase in the episodes of pneumonia, but that hospital stay was significantly prolonged as compared to patients not treated with steroids, resulting in an average increase of US\$51,504 in hospitalization costs per admission. ${ }^{23}$

\section{Reproducibility}

The ultimate test though of a clinical trial is reproducibility - the ability of other researchers to generate the same results when the same protocols are followed in different settings. This is a critical point in evaluating the NASCIS results, since the raw data was only released several years after the study, and as Coleman ${ }^{19}$ noted:

"The numbers, tables, and figures in the published reports are scant and inconsistently defined, making it impossible even for professional statisticians to duplicate the analysis, to guess the effect of changes in assumptions, or to supply the missing parts of the picture..."

Aside from the NASCIS trials, the TraumaBank Informational Repository currently lists two prospective and 7 retrospective trials dealing with the issue of steroids for cord injury. ${ }^{12}$

Among the prospective trials, Otani ${ }^{24}$ studied 158 patients in a randomized, un-blinded trial using the $24 \mathrm{MP}$ protocol drawn up by NASCIS. He reported trends towards increased infectious complications and improved sensation in the group given methylprednisolone. However, the exclusion of 41 patients after randomization (primarily for protocol violations) makes it difficult to consider this trial as Level I evidence.

Pettijean and Pointillart ${ }^{25}$ conducted a randomized, double-blinded trial of 106 patients, testing the 24MP protocol, as well as the use of nimodipine and a methylprednisolone-nimodipine combination. After 1 year, no significant enhancement of ASIA scores could be attributed to the use of methylprednisolone (or to the use of nimodipine, or to their center's policy of early surgical intervention).
The 5 retrospective trials listed by TraumaBank do not support the use of steroids either. Gerhardt ${ }^{26}$ compared Frankel scores of 188 patients treated under the NASCIS protocols with 175 treated prior to their advent. Ninety patients in the latter group received no steroids at all. No significant improvement was found among patients who received steroids. George ${ }^{27}$ reviewed 130 patients, 75 of whom were treated with the NASCIS protocols. No significant improvement in neurologic outcome was noted in the methylprednisolone group, although they manifested a non-significant trend towards increased infectious complications. Poynton ${ }^{28}$ reviewed 71 consecutive patients, with those patients received more than 8 hours after trauma serving as the control group and not receiving steroids. No difference in ASIA scores were seen in the patients who had received methylprednisolone. Of interest is that in both the George and Poynton studies, equivalent outcomes were seen in both the control and steroid groups despite the control groups being older and more severely injured on admission to the studies.

Penetrating injuries to the spine have always been considered a special category of injury, and here, the use of steroids has also been reviewed. Prendergast, ${ }^{29}$ Levy $^{30}$ and Heary $^{31}$ retrospectively reviewed their experience with steroids in the treatment of penetrating injuries to the spine. None of these studies showed improved neurological outcome associated with either methylprednisolone or dexamethasone, with Heary further documenting a significant increase in gastritis in patients given dexamethasone, and pancreatitis in those receiving methylprednisolone. Prendergast also found a poorer neurologic outcome in patients with penetrating injuries who were given steroids. Gerndt ${ }^{22}$ conducted a retrospective review focused on the incidence of infectious complications. He compared 91 patients who had received methylprednisolone to a historical control of 140 patients treated without steroids. The use of steroids was associated with a significant increase in the incidence of early pneumonia, as well as prolonged ICU confinement and ventilator dependence. There was, however, no difference noted in the long-term outcomes.

Shor $^{20}$ reviewed the same studies mentioned above, following the guidelines recommended by the Evidence Based Working Group in Canada, and arrived at a similar conclusion:

"...this systemic review does not support the use of high dose methylprednisolone in acute spinal cord injury to improve neurological recovery. A deleterious effect on early mortality and morbidity cannot be excluded..."

\section{Current Attitudes}

The absence of clear data showing a benefit in the use of steroids for acute spinal cord injury has led to a significant decrease in the prevalence of its use. The American 
Association of Neurological Surgeons and the Congress of Neurological Surgeons stated in their influential 2002 Guidelines for the Management of Acute Cervical Spine and Spinal Cord Injuries: ${ }^{21}$

"Treatment with methylprednisolone for either 24 or 48 hours is recommended as an option in the treatment of patients with acute spinal cord injuries that should be undertaken only with the knowledge that the evidence suggesting harmful side effects is more consistent than any suggestion of clinical benefit..."

A study in Canada ${ }^{32}$ showed that $76 \%$ of spine surgeons surveyed in 2003 prescribed methyprednisolone for acute spinal cord injury. In response to the same questionnaire in 2008, 76\% now answered they did not administer methylprednisolone, and of the physicians who continued to use it, one-third did so it due to fear of litigation. At the request of the Canadian Spine Society and the Canadian Neurosurgical Society, the Canadian Association of Emergency Physicians reviewed the evidence behind the use of methylprednisolone and came out with a position statement saying:

"Methylprednisolone for acute spinal cord injury is not a standard of care; it only a treatment option." 33 and further:

"Physicians should not feel intimidated into prescribing highdose methylprednisolone for acute spinal cord injuries...(it) has not been adequately tested..."

This point of view was re-iterated by the Canadian Medical Association. ${ }^{34}$

In Switzerland, during the period 2001 to 2003, 96\% of patients with neurological deficit after spinal cord injury were treated with high dose methylprednisolone. This dropped to $23 \%$ in the period 2008 to 2010 . Pooled data showed no statistical improvement in neurological recovery between the two groups. ${ }^{35}$ This experience was reflected in a report from Japan ${ }^{36}$ where a 2 year period in which all cervical spinal cord injury patients were treated according to the NASCIS protocols was followed by a 2 year period in which the use of steroids had been abandoned. No significant difference in neurologic recovery was noted between the two groups.

\section{Summary}

This article reviewed the controversy over the use of steroids in the treatment of acute injury to the spinal cord. The theoretical foundation for this practice was largely based on the results of the National Acute Spinal Cord Injury Study, a large, multi-center trial that was considered a model research protocol at that time. Questions over the research design and statistical analysis as well as equivocal benefits seen in trials under other groups have led to a dilution of the impact of their recommendations to the point where use of steroids in cord injury is seen as an option of treatment with equivocal benefits that must be balanced against significant risk of complications.

\section{References}

1. Mosier versus Southern California Physicians Insurance Exchange. 63 California Appelate 4th 1022, 74 California Rptr. 2 d 550, 1998.

2. Hurlbert RJ. Methylprednisolone for acute spinal cord injury: an inappropriate standard of care. J Neurosurg. 2000; 93(1 Suppl):1-7.

3. Congress of Neurological Surgeons Education Committee. Medical Student Curriculum in Neurosugery: Diagnosis and management of spinal cord injury [Online]. 2013 [cited 2013 May]. Available from http://w3.cns.org/education/medStudCur/index2.asp

4. Bracken MB. Pharmacological interventions for acute spinal cord injury. Cochrane Database Syst Rev. 2000; (2):CD001046.

5. Bracken MB. Steroids for acute spinal cord injury. Cochrane Database Syst Rev. 2012; 1:CD001046.

6. Bracken MB, Collins WF, Freeman DF, et al. Efficacy of methylprednisolone in acute spinal cord injury. JAMA. 1984; 251(1):4552.

7. Bracken MB, Shepard MJ, Hellenbrand KG, et al. Methylprednisolone and neurological function one year after spinal cord injury. Results of the National Acute Spinal Cord Injury Study. J Neurosurg. 1985; 63(5):704-13.

8. Bracken MB, Shepard MJ, Collins WF, et al. A randomized, controlled trial of methylprednisolone or naloxone in the treatment of acute spinalcord injury. Results of the second National Acute Spinal Cord Injury Study. N Engl J Med. 1990; 322(20):1405-11.

9. Bracken MB, Shepard MJ, Collins WF, et al. Methylprednisolone or naloxone treatment after acute spinal cord injury: 1-year follow-up data. Results of the second National Acute Spinal Cord Injury Study. J Neurosurg. 1992; 76(1):23-31.

10. Bracken MB, Shepard MJ, Holford TR, et al. 1997. Administration of methylprednisolone for 24 or 48 hours or tirilazad mesylate for 48 hours in the treatment of acute spinal cord injury: Results of the Third National Acute Spinal Cord Injury Randomized Controlled Trial National Acute Spinal Cord Injury Study. JAMA. 1997; 277(20):1597-604.

11. Bracken MB, Shepard MJ, Holford TR, et al. Methylprednislone or tirilazad mesylate administration after acute spinal cord injury: 1-year follow up. Results of the third National Acute Spinal Cord Injury randomized controlled trial. J Neurosurg. 1998; 89(5):699-706.

12. Brohi K. TraumaBank Informational Repository: Steroids for Spinal Cord Injury [Online]. 2005 [cited 2013 May]. Available from http://www.trauma.org/archive/traumabank.html

13. Black P, Markovitz RS. Experimental spinal cord injury in monkeys: comparison of steroids and local hypothermia. Surg Forum. 1971; 22:409-11.

14. Campbell JB, DeCrescito V, Tomasula JJ, Demopoulos HB, Flamm ES, Ransohoff J. Experimental treatment of spinal cord contusion in the cat. Surg Neurol. 1973; 1(2):102-6.

15. Lewin MG, Hansebout RR, Pappius HM. Chemical characteristics of traumatic spinal cord edema in cats. J Neurosurg. 1974; 40(1):65-75.

16. Eidelberg E, Staten E, Watkins CJ, Smith JS. Treatment of experimental spinal cord injury in ferrets. Surg Neurol. 1976; 6(4):243-6.

17. Bracken MB. In "Comments", Guidelines for the management of acute cervical spine and spinal cord injuries. Neurosurgery. 2002; 50(3):S-iv-Sxx.

18. Hanigan WC, Anderson RJ. Commentary on NASCIS-2. J Spinal Disord. 1992; 5(1):125-31.

19. Coleman WP, Benzel D, Cahill DW, et al. A critical appraisal of the reporting of the National Acute Spinal Cord Injury Studies (II and III) of methylprednisolone in acute spinal cord injury. J Spinal Disord. 2000; 13(3):185-99.

20. Short DJ, El Masry WS, Jones PW. High dose methylprednisolone in the management of acute spinal cord injury - a systematic review from a clinical perspective. Spinal Cord. 2000; 38(5):273-86.

21. Pharmacological therapy after acute cervical spinal cord injury. In "Guidelines for the management of acute cervical spine and spinal cord injuries. Neurosurgery. 2002; 50(3 Suppl):S63-72.

22. Gerndt S, Rodriguez JL, Pawlik JW, et al. Consequences of high-dose steroid therapy for acute spinal cord injury. J Trauma. 1997; 42(2):279-84. 
23. Galandiuk S, Raque G, Appel S, Polk HC Jr. The two-edged sword of large-dose steroids for spinal cord trauma. Ann Surg. 1993; 218(4):41927.

24. Otani K, Abe H, Kadoya S, et al. Beneficial effect of methylprednisolone sodium succinate in the treatment of acute spinal cord injury. Sekitsui Sekizui J. 1994; 7:633-47.

25. Pointillart V, Pettijean ME, Wiart L, et al. Pharmacological therapy of spinal cord injury during the acute phase. Spinal Cord. 2000; 38(2):71-6.

26. Gerhart KA, Johnson RL, Menconi J, Hoffman RE, Lammertse DP. Utilization and effectiveness of methylprednisolone in a populationbased sample of spinal cord injured persons. Paraplegia. 1995; 33(6):31621.

27. George ER, Scholten DJ, Buechler CM, Jordan-Tibbs J, Mattice C, Albrecht RM. Failure of methylprednisolone to improve the outcome of spinal cord injuries. Am Surg. 1995; 61(8):659-64.

28. Poynton AR, O'Farrel DA, Shannon F, Murray P, McManus F, Walsh MG. An evaluation of the factors affecting neurological recovery following spinal cord injury. Injury. 1997; 28(8):545-8.

29. Prendergast MR, Saxe E, Ledgerwood AM, et al. Massive steroids do not reduce the zone of injury after penetrating spinal cord injury. J Trauma. 1994; 37(4):576-9.
30. Levy ML, Gans W, Wijesingh HS, SooHoo WE, Adkins RH, Stillerman $\mathrm{CB}$. Use of methylprednisolone as an adjunct in the management of patients with penetrating spinal cord injury: outcome analysis. Neurosurgery. 1996; 39(6):1141-9.

31. Heary RF, Vaccaro AR, Mesa JJ, et al. Steroids and gunshot wounds to the spine. Neurosurgery. 1997; 41(3):576-84.

32. Hurlbert RJ, Hamilton MG. Methylprednisolone for acute spinal cord injury: 5-year practice reversal. Can J Neurol Sci. 2008; 35(1):41-5.

33. Canadian Association of Emergency Physicians. CAEP Position statement: Steroids in acute spinal cord injury. CJEM. 2003; 5(1):7-9.

34. Hugenholtz H. Methylprednisolone for acute spinal cord injury: not a standard of care. CMAJ. 2003; 168(9):1145-6.

35. Felleiter P, Muller N, Schumann F, Felix O, Lierz P. Changes in the use of the methylprednisolone protocol for traumatic spinal cord injury in Switzerland. Spine (Phila Pa 1976). 2012; 37(11):953-6.

36. Ito $\mathrm{Y}$, Sugimoto $\mathrm{Y}$, Tomioka M, Kai N, Tanaka M. Does high dose methylprednisolone sodium succinate really improve neurological status in patient with patient with acute cervical cord injury? A prospective study about neurological recovery and early complications. Spine (Phila Pa 1976). 2009; 34(20):2121-4.

\section{UPM's VPN Service Pilot Testing}

We are pleased to announce the pilot testing of UPM's VPN service.

Virtual Private Networking (VPN) allows users to connect securely to UP Manila IT resources especially access to online journals and e-book subscriptions of the Library from a remote location.

For the purpose of pilot testing, UP Manila libraries are accepting individual request with a UPM Affiliation and one of the following types: Faculty / Students / Employees. Please apply for a VPN account at your respective college/unit libraries. For PGH personnel, apply at the Medical Library.

VPN client software are available for the following operating systems: Windows 7 / Vista / XP, Mac OS, and Ubuntu

Also available are VPN configurations for iPhones and iPads. Android devices are not yet supported.

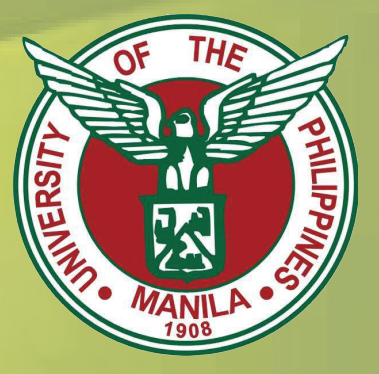

